\title{
Mises en scène et mémoires de la consécration de l'église dans l'Occident médiéval
}

Centre d'études médiévales d'Auxerre, 27-29 juin 2005

\section{Didier Méhu}

\section{(2) OpenEdition}

\section{Journals}

Édition électronique

URL : https://journals.openedition.org/cem/372

DOI : 10.4000/cem.372

ISSN : 1954-3093

Éditeur

Centre d'études médiévales Saint-Germain d'Auxerre

Édition imprimée

Date de publication : 15 août 2006

ISSN : 1623-5770

\section{Référence électronique}

Didier Méhu, « Mises en scène et mémoires de la consécration de l'église dans l'Occident médiéval », Bulletin du centre d'études médiévales d'Auxerre | BUCEMA [En ligne], 10 | 2006, mis en ligne le 08 septembre 2006, consulté le 22 septembre 2022. URL : http://journals.openedition.org/cem/372 ; DOI : https://doi.org/10.4000/cem.372

Ce document a été généré automatiquement le 22 septembre 2022.

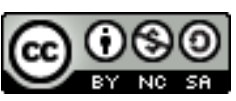

Creative Commons - Attribution - Pas d'Utilisation Commerciale - Partage dans les Mêmes Conditions 4.0 International - CC BY-NC-SA 4.0

https://creativecommons.org/licenses/by-nc-sa/4.0/ 


\title{
Mises en scène et mémoires de la consécration de l'église dans l'Occident médiéval
}

Centre d'études médiévales d'Auxerre, 27-29 juin 2005

\author{
Didier Méhu
}

1 Une vingtaine de médiévistes se sont réunis au Centre d'études médiévales d'Auxerre du 27 au 29 juin 2005 pour réfléchir aux implications sociales de la consécration de l'église au Moyen Âge central ${ }^{1}$. En mettant l'accent sur les «mises en scène » et les «mémoires » de la consécration, il s'agissait d'envisager conjointement les différents modes de réification de la cérémonie à une période où celle-ci a fait l'objet de normalisations et de nombreux commentaires.

2 L'historien de la consécration de l'église n'est pas dépourvu de matériaux : des textes (ordines, historiae, sermons, exégèse, formules de bénédiction, oraisons...), des images peintes et sculptées, des églises, des inscriptions dans les églises, des pièces musicales (antiennes, tropes, proses...), autant «d'objets » qui articulent par le verbe et par l'image des éléments considérés comme probants pour transmettre le souvenir d'une consécration. Le point de départ était de considérer ces «objets» moins comme des «sources» dont jaillirait une quelconque vérité objective que comme des discours rigoureusement construits qui évoquent la naissance d'une église au moment de sa consécration et la (re)naissance du corps social qui lui est lié. Pour ce faire, les participants étaient invités à dépasser l'analyse typologique et sectorielle des documents qui tend à isoler, par exemple, la genèse des «textes liturgiques » de celle des formes narratives qui évoquent la consécration, ou à étudier de manière isolée les images, les inscriptions et les tropes de consécration. Il s'agissait également d'articuler les réflexions sur l'image, l'écrit, le sensoriel, le gestuel pour comprendre conjointement le déroulement des cérémonies, les productions «mémorielles" auxquelles elles ont donné naissance et leurs effets sociaux. Il fallait enfin envisager le volet institutionnel de la consécration. Si la liturgie transforme symboliquement le lieu ecclésial, la sanctio qui le situe désormais hors du monde profane assure la pérennité de 
la transformation en générant des interdits et de nouvelles hiérarchies sociales et spatiales.

3 En amont, il était nécessaire de réfléchir sur le champ sémantique de la consécration: consecratio et dedicatio sont traditionnellement présentés comme des termes synonymes que les auteurs utilisaient l'un pour l'autre et que les historiens ne distinguent généralement pas. Et l'on ne saurait réduire l'enquête à ces deux mots dans la mesure où un troisième terme est très souvent employé, benedictio, d'autant plus intéressant qu'à la différence des deux autres il semble inconnu du latin classique. L'entreprise n'est pas une mince affaire tant les occurrences sont nombreuses et la matière apparemment impossible à traiter avec les outils actuels de la statistique lexicale. Ce semi constat d'échec souligné par Alain GUERREAU ne doit pas pour autant décourager les recherches qui, sans doute, devront se concentrer sur une période restreinte, voire une sélection de documents ${ }^{2}$.

4 Les recherches menées récemment sur les objets, lieux et territoires sacrés et sur les conditions de production des discours sur le lieu consacré (normatif, exégétique, historiographique et pastoral) étaient considérées comme un point de départ pour les discussions ${ }^{3}$. Sur cette base, les débats ont d'abord concerné le déroulement du rituel (Didier MÉHU, «La dynamique du rituel de consécration de l'Église, VIII -XIII ${ }^{e}$ siècle »). Le dépôt des reliques dans l'autel et la célébration de la première eucharistie sont les deux événements fondateurs et les deux seules opérations indispensables depuis l'Antiquité tardive. Elles sont solennisées par des gestes et des paroles que les ordines pour la consécration de l'église indiquent avec de plus en plus de précision depuis leur première formulation articulée au début du viII ${ }^{e}$ siècle jusqu'aux grandes compilations de la fin du xiII dont le pontifical de Guillaume Durand est le point d'orgue. Le souci croissant de précision attesté par les ordines peut se lire comme le reflet d'une volonté d'uniformiser les rituels au sein de l'Église romaine et de fournir aux célébrants un instrument pratique pour conduire leur office, mais ce serait considérer uniquement la valeur normative des codifications liturgiques. Or, il est clair que celles-ci ne sont pas simplement des marches à suivre, mais également des sortes de miroirs de l'Église qui témoignent de l'articulation progressive de la réflexion ecclésiologique autour du magistère épiscopal et du lieu de culte. La théâtralisation grandissante de la consécration et le souci de commenter chaque geste pour exprimer ses significations implicites manifestent un degré de conscientisation aigu du rôle transitoire de la cérémonie et du lieu lui-même que l'on montre comme l'antichambre du paradis. Il s'agit de montrer qu'un lieu profondément humain - l'église, un édifice de pierres et de chaux élevé par les maçons et les produits de l'exploitation terrestre - est en train de devenir le locus terribilis propice à tous les transitus de la terre vers le ciel. Cela s'effectue de plusieurs manières. Le célébrant joue le rôle central de l'opérateur de sacralité. Avec son pouce et sa main, il mêle l'eau, le vin, le sel et les cendres pour en faire des " espèces transitoires ", il imprime les signes de croix sur l'autel et les murs de l'église, il oint l'autel et porte les reliques. Par ses déplacements corporels, il exprime la sacralité du lieu en tournant plusieurs fois autour de l'autel, à l'intérieur et à l'extérieur du bâtiment. Le verbe se joint aux gestes pour signifier la transformation, tant par les formules de bénédiction que par le sermon prononcés par le célébrant, et par les antiennes, répons et proses entonnés par le chœur. Le peuple, spectateur majoritairement passif, n'assiste visuellement qu'à une partie de la cérémonie. Sa condition ne lui permet pas encore de participer à la vision des mystères, il est 
cantonné dans le rôle du donateur, du constructeur et de l'auditeur du verbe épiscopal qui donne un sens à ses actions.

5 En se fondant principalement sur l'ordo ad benedicendam ecclesiam (Pontifical romanogermanique - PRG 40) et sur le long commentaire qui le précède dans la plupart des manuscrits, Quid significent duodecim candelae (PRG 35), Catherine GAUTHIER a envisagé les fonctions de l'encens et du luminaire au cours de cinq étapes importantes de la dédicace de l'église: la translation des reliques la veille de la consécration, l'illumination des douze chandelles au début de la cérémonie, les onctions de l'autel, le dépôt des reliques dans la confessio et l'illumination de l'église avant la première messe (C. Gauthier, «L'encens et le luminaire dans les dédicaces d'églises au haut Moyen Âge »). L'usage de l'encens n'est évidemment pas propre à la consécration et l'on peut aisément le rapprocher de ceux connus pour d'autres rituels. Sa présence dans la translation des reliques s'avère ainsi un signe de révérence envers le saint qui n'est pas sans évoquer son usage propitiatoire lors des funérailles. Mais c'est surtout à l'intérieur de l'église que l'encens accompagne la consécration. Les onctions de l'autel ne se conçoivent pas sans lui, contrairement aux aspersions qui semblent s'en dispenser. L'encens brûlé est porté en forme de croix au-dessus de l'autel avant les onctions, puis tout autour de l'autel lorsque le célébrant oint la table. Il s'agit alors de purifier le lieu (l'odeur, la croix) et de le séparer du monde profane (le cercle) afin de le rendre apte à accueillir les offrandes. Les ordines évoquent également deux usages de l'encens qui semblent spécifiques à la consécration : la disposition en croix sur la table d'autel de grains d'encens allumés (à partir du XII ${ }^{e}$ siècle) et le dépôt de trois grains d'encens non brûlés dans la confessio avec les reliques. L'usage de l'encens brûlé à même la table d'autel pouvait être vu comme le marquage du sceau divin à l'aide d'une species dont les vertus transitoires étaient particulièrement soulignées (comme l'huile des onctions). Le dépôt des grains non brûlés dans la confessio, non glosé dans le PRG 35, tient sans doute aux vertus olfactives de la résine et à ses usages dans l'embaumement des corps. Jusqu'au $\mathrm{XII}^{\mathrm{e}}$ siècle, les ordines indiquent que l'on déposait également trois hosties consacrées dans la confessio du saint, ce qui l'associait implicitement au sépulcre du Christ. Par ailleurs, l'introduction du luminaire dans l'église renvoie à la théologie de la lumière et à ses matérialisations multiples au sein de l'architecture religieuse médiévale. En encadrant la consécration par deux rituels de la lumière (l'installation de douze chandelles en cercle dès l'entrée de l'évêque dans l'église) et l'illumination complète de l'église lors de l'incipit de la messe, on soulignait le baptême du lieu de culte, désormais annonciateur de la Bonne nouvelle (les douze chandelles étant associées aux apôtres), inscrit dans le réseau de l'Église universelle et apte à recevoir la lumière divine pour conduire le cheminement spirituel des fidèles.

Les ordines et leurs commentaires ne sont pas les seuls moyens d'exprimer la transformation de l'église et pour que la réification soit complète il importe que l'on se souvienne du moment fondateur marqué par la consécration. L'invitation à commémorer annuellement la fête de la dédicace prend ici tout son sens d'autant qu'elle doit être formulée, selon les ordines, dans le sermon épiscopal qui clôt la consécration ${ }^{4}$. L'écrit et l'image prennent ensuite le relais pour inscrire la consécration dans la mémoire de la communauté ecclésiale.

7 Partant du récit de la consécration de l'église Sainte-Agathe de Rome par Grégoire le Grand en 591/592, Martin RocH a montré comment le thème biblique de la bonne odeur liée à la théophanie et de la mauvaise odeur liée aux surgissements diaboliques était 
réarticulé dans plusieurs récits évoquant le lieu ecclésial et sa consécration ("Théophanie et liturgie: les odeurs de la dédicace de l'église Sainte-Agathe selon Grégoire le Grand »). Dans le livre III de ses Dialogues, Grégoire rapporte les événements miraculeux survenus lors de la consécration de l'église Sainte-Agathe, ancienne église des Ariens. Alors que l'on célébrait la première messe dans l'église consacrée, les fidèles présents purent sentir (sentire, c'est-à-dire percevoir de manière sensorielle) un porc qui s'enfuyait de l'église en se faufilant entre eux. Le lendemain, une nuée miraculeuse qui descendit du ciel sur l'autel emplit totalement le lieu en dégageant un parfum suave. La relation discursive de ces deux événements insiste sur l'opposition entre l'animal profondément charnel et malodorant qu'est le porc - évoquant implicitement les Ariens hérétiques - et la nuée spirituelle qui sacralise le lieu en empêchant quiconque d'y pénétrer. Les propos du pape font explicitement référence à la nuée divine qui emplit le Temple de Salomon au moment de sa dédicace, mais il s'inscrit aussi dans les pratiques et les discours liturgiques contemporains (ordines, bénédictions et formulaires de messe de dédicace) qui associent la consécration à un baptême et un exorcisme. La nuée odorante suggère en effet l'olfaction de l'autel par les onctions parfumées et les encensements, ou l'antienne de la chrismation : Ecce odor filii. D'autres histoires plus ou moins contemporaines du récit de Grégoire - depuis l'Histoire de la persécution des Vandales en Afrique par Victor de Vita (488/489) jusqu'aux récits hagiographiques anglais de la fin du vIII ${ }^{\mathrm{e}}$ siècle, en passant par les Histoires de Grégoire de Tours - montrent quelques constantes dans l'usage discursif des bonnes odeurs, que l'on associe à la consécration de l'église, à l'œuvre bienfaitrice d'un évêque, à la présence d'un saint ou à la messe.

Dans les Dialogues, le récit exemplaire de la consécration contribue à montrer la présence agissante de Dieu par l'intermédiaire de l'un de ses plus zélés protagonistes. La valorisation peut également positionner une église ou la communauté de ses desservants dans la geste de l'histoire du salut. À Saint-Étienne de Dijon, le récit prend place au cœur d'une construction historiographique exemplaire (Laurent DURNECKER, "Consécrations d'autels et dépôts de reliques. L'exemple de Saint-Étienne de Dijon du $\mathrm{XI}^{\mathrm{e}}$ au début du XIII ${ }^{\mathrm{e}}$ siècle »). L'église Saint-Etienne de Dijon est une collégiale de chanoines réformée au début du XII siècle. Incendiée en 1137, elle est reconstruite rapidement et la renaissance s'affirme par la consécration de nouveaux autels et la composition de deux dossiers mémoriels : un cartulaire (v. 1141-1162) et la vie de Garnier de Mailly (v.1153-1170), le prévôt du chapitre à qui l'on attribue la construction de la collégiale au milieu du $\mathrm{XI}^{\mathrm{e}}$ siècle. Dans le cartulaire, le récit des consécrations se présente comme une conclusion à la série de chartes et de bulles pontificales qui attestent des droits des chanoines sur des terres environnantes. On peut y voir un moyen de souligner les prérogatives monastiques face aux revendications des seigneurs locaux et à l'affirmation du dominium du duc de Bourgogne sur la ville de Dijon, mais aussi le rappel de la nécessaire dotation des lieux consacrés. Associé à la vie de Garnier de Mailly, le même récit augmenté de deux mentions relatives à des consécrations survenues en 1157/58 et 1170 souligne le rôle fondateur du prévôt du XI ${ }^{\mathrm{e}}$ siècle. La structure du récit est elle-même significative. La trame chronologique est délaissée au profit d'une relation discursive entre la destruction (incendie) et la renaissance (consécrations), des liens directs sont tracés entre les deux "naissances" par la mention d'un transfert de reliques entre l'ancien maître autel et le nouveau, et le récit précise soigneusement les vocables des autels. L'église Saint-Étienne apparaît ainsi comme un panthéon fondé sur l'accumulation de 
plusieurs fragments de reliques qui dessinent une réduction de l'Église universelle dans ses dimensions spatiales et temporelles, sans négliger le contexte local (saints du diocèse de Langres) et communautaire (saints des communautés religieuses associées à Saint-Étienne).

Dans le sillage des réflexions de Philippe Buc ${ }^{5}$, Louis HAMiLton nous invite à considérer avec prudence les «sources » qui évoquent des rituels dans la mesure où les textes liturgiques comme les narrations de la consécration (récits ou images) semblent moins témoigner du déroulement précis de celles-ci que de la manière dont les différents groupes sociaux ont tenté de les contrôler ("The dangers of Ritual in Eleventh-Century Italy : Between Liturgical Texts and Historical Records »). Force est de constater, tout d'abord, que les pontificaux italiens des $\mathrm{XI}^{\mathrm{e}}$ et $\mathrm{XII}^{\mathrm{e}}$ siècles présentent des différences sensibles relativement au déroulement de la consécration, y compris pour des étapes qui peuvent paraître essentielles comme l'onction de l'autel, l'aspersion des murs de l'église ou les circuits autour de celle-ci. Dans certains cas, la topographie du site ou l'inachèvement de la construction impliquaient des aménagements notables et l'historien est alors bien en peine de préciser le déroulement de la cérémonie. Cette absence d'uniformité liturgique ne semble pas avoir nui à la multiplication des consécrations ni à la surenchère sur leurs significations symboliques, parfois contradictoires. Les modalités de la translation du corps de saint Giminiano et de la consécration de l'autel sous lequel il reposait dans la cathédrale de Modène, en 1106, donna lieu à des contestations violentes entre l'évêque et le peuple de la cité au moment où naissaient les premières revendications communales. Le point d'achoppement se situait dans la possibilité de voir les reliques au moment de la translation, ce que l'évêque refusait au peuple. Un compromis fut trouvé grâce à l'arbitrage de la comtesse Mathilde de Canossa, de l'évêque de Reggio et du pape Pascal II, et la consécration de l'autel fut l'occasion de redéfinir les hiérarchies sociales et les compétences respectives des différents corps constitutifs de la ville. Un manuscrit de la seconde moitié du XIII ${ }^{\mathrm{e}}$ siècle l'exprima par le verbe et l'image en articulant la translation des reliques, leur dévoilement, le compromis des autorités, les présents effectués, le rôle défensif des custodes monumenti -chevaliers et citoyens-, et la construction de la cathédrale par le peuple sous la conduite d'un architector.

Le dossier provençal, envisagé par Michel LAUwERs et Yann CODOU, permet d'articuler étroitement deux modes de commémoration écrite de la consécration, la charte et l'inscription monumentale («Marques de dédicace et récits de consécration des églises provençales, $\mathrm{XI}^{\mathrm{e}}-\mathrm{XII}{ }^{\mathrm{e}}$ siècles »). La rédaction de chartes de consécration s'inscrit dans une pratique valorisée dès la période carolingienne par laquelle l'évêque recommandait de consigner par écrit les promesses de donations qui devaient précéder ou accompagner la (re)construction d'une église. Nombreuses en Catalogne dès le $\mathrm{Ix}^{\mathrm{e}}$ siècle, elles se développent en Provence au milieu du $\mathrm{xl}^{\mathrm{e}}$ lors de la diffusion de la réforme visant notamment à restituer aux évêques des églises et leurs revenus possédés par des laïcs. Une vingtaine d'actes conservés en original ou par une copie dans le grand cartulaire de Saint-Victor de Marseille (vers 1080) montre le réseau des évêques provençaux (Arles, Aix, Nîmes, Apt) et des moines victorins engagés dans une telle politique qui se veut une régénération sociale. Les dispositifs des chartes évoquent les aspects juridiques de la consécration et, dans une moindre mesure, ses aspects liturgiques : la vérification de la dotation suffisante de l'église, l'accord de l'évêque et sa venue sur place pour procéder au rituel en présence des principaux représentants de 
l'aristocratie régionale, la consécration du lieu par le transfert des reliques, les aspersions, les bénédictions et la délimitation d'un atrium. Les préambules, parfois fort développés, témoignent de la réflexion qui s'élaborait alors autour du rituel pour en valoriser les aspects symboliques et justifier les nouvelles pratiques sociales qu'il générait. La dotation de l'église est présentée comme un sponsalicium justifié par les noces du Christ et de l'ecclesia, la consécration de l'église est nécessaire parce qu'elle est le «lieu du véritable sacrifice » et l'incarnation du Christ dans le monument s'inscrit dans l'histoire du salut dans la mesure où le Christ lui-même et les apôtres sont présentés comme les premiers fondateurs et constructeurs d'églises. Le discours des chartes et leur articulation dans le cartulaire permet ainsi de construire une nouvelle histoire, polarisée par l'événement fondateur de la consécration et par les personnages emblématiques des donateurs-constructeurs-consécrateurs que sont les évêques, les moines et les aristocrates.

11 Se concentrant sur les monuments provençaux de la période romane, Yann codou a envisagé la dialectique entre les inscriptions durables et fugaces dans le bâtiment ecclésial en relation avec la consécration. Les ordines évoquent plusieurs pratiques d'inscription éphémères sur les parties constitutives de l'édifice, qu'il s'agisse d'inscription de lettres (tracé des alphabets grec et latin sur le sol de la nef) ou de signes (onctions en forme de croix sur les murs intérieurs, la table d'autel et la confessio). L'observation des bâtiments montre des pratiques d'inscription qui ne sont pas évoquées dans les ordines mais qui relèvent du souci de marquer durablement le souvenir de la consécration dans la structure monumentale. Aux croix éphémères tracées sur les autels et les murs s'ajoutent des croix peintes (mentionnées dans les ordines dès le XIII ${ }^{\mathrm{e}}$ siècle), mais aussi des croix sculptées de part et d'autre du portail (cathédrale de Vaison) ou sur les murs intérieurs de l'église, chaque croix étant inscrite dans un cercle (Valbonne). L'alphabet a pu faire l'objet d'une véritable inscription monumentale comme dans l'église de Beaumont de Ventoux (deuxième moitié du XII ${ }^{\mathrm{e}}$ siècle) où l'on a gravé un triple alphabet sur les murs extérieurs du bâtiment, sur la même assise, en partant de la porte méridionale et en tournant dans le sens inverse des aiguilles d'une montre. Cet alphabet circulaire a une valeur commémorative dans la mesure où il évoque le triple circuit effectué par l'évêque lors de l'aspersion des murs extérieurs de l'église, mais on peut le lire également comme un moyen de souligner les fonctions symboliques de l'édifice en rappelant qu'il est le lieu à partir duquel s'articule l'intellection verbale du monde.

12 Les relations étroites entre consécration et inscription s'expriment parfois de manière inattendue. Marie-José GASSE-GRANDJEAN s'est penchée sur un acte tout à fait original conservé dans le fonds du prieuré de Xures aux Archives de la Moselle («La charteinscription de la chapelle de Xures»). Il s'agit d'un parchemin de 54 sur $52 \mathrm{~cm}$ sur lequel est copié, au recto, en grosses lettres capitales à la graphie soignée, un bref texte qui évoque la consécration de la chapelle de Xures en 1072. L'énoncé est simple. On retient la date, le nom du consécrateur, le patronage de la chapelle et la liste des reliques déposées. Au verso du même parchemin est copié un acte de douaire daté de l'an 1022 par lequel un certain Richard constitue la dot de sa fiancée Cunégonde. Celleci compta ultérieurement parmi les donateurs qui permirent de constituer la dotation de la chapelle de Xures, ce qui éclaire l'articulation entre les deux côtés du parchemin. La « charte » de consécration n'en demeure pas moins étrange. La forme des lettres, la teneur de l'acte et l'absence de signe de validation l'apparentent aux inscriptions 
monumentales et il est probable qu'elle soit la copie d'une inscription aujourd'hui perdue. L'analyse paléographique de la charte plaide pour une copie de l'inscription à la fin du XII ${ }^{\mathrm{e}}$ siècle, une manière de contribuer à la rédaction de l'histoire du prieuré un siècle après la consécration de l'église. Cette hypothèse souligne l'importance des pratiques d'écriture en relation avec la consécration, tant pour souligner des solennités liturgiques que pour valoriser l'histoire d'une église.

13 Aussi importe-t-il de prendre en compte le rôle des lettres dans la sacralité de l'édifice - et plus globalement le rôle du verbe comme vecteur pour connaître et représenter le divin. C'est à une telle ouverture que nous invite Cécile TREFForT en situant les inscriptions de consécration « informatives » (date, nom du célébrant et des donateurs) parmi l'ensemble des textes inscrits dans le monument et qui participent à sa sacralité ("Une consécration "à la lettre": place, rôle et autorité des textes inscrits dans la sacralisation de l'édifice »). Dans cette perspective, on peut considérer les inscriptions invisibles, qu'elles soient éphémères (alphabet) ou cachées (sur la première pierre de l'église ensuite enfouie dans les fondations, ou au revers d'une pierre de parement), comme des moyens d'imprimer la présence du verbe dans le bâtiment sans nécessairement passer par la visibilité d'un texte écrit. Par leur caractère durable, les inscriptions visibles, peintes ou gravées, situent l'édifice dans le temps: un temps humain en rappelant le moment de la consécration, le nom des prélats consécrateurs, de fondateurs et des donateurs; un temps eschatologique en rappelant de manière durable le statut de l'édifice, en impliquant parfois la communauté des fidèles interpellés par l'inscription pour prier pour le salut des fondateurs/consécrateurs ; un temps cyclique qui insiste sur la date de la consécration en incitant implicitement à la commémorer chaque année. Sans oublier l'inscription de versets qui évoquent la présence divine dans le lieu et son statut transitoire (domus domini et porta coeli) tout en effectuant un lien implicite avec sa consécration dans la mesure où ces phrases sont utilisées comme antiennes ou répons lors du rituel. La réflexion sur la portée de ces textes ne saurait faire abstraction de leur lieu d'inscription. La répartition des inscriptions dans l'église peut en effet se lire comme l'expression d'un espace ecclésial polarisé (inscriptions sur l'autel), structuré (les portes, les colonnes) et articulé (inscriptions sur l'autel qui renvoient à la consécration de l'église, ou sur un mur pour évoquer la consécration de l'autel); l'articulation pouvant se lire à l'intérieur de l'édifice ou dans un cadre géographique large, celui de la ville (inscription dans une église qui évoque la consécration d'autres lieux de culte de la ville), voire de l'Église (noms des prélats présents, voire du pape).

14 La réflexion sur la visibilité/invisibilité des inscriptions nous conduisait nécessairement à une réflexion sur les images liées à la consécration du bâtiment ecclésial. Cependant, l'objet figuré « image de consécration » n'est pas mieux défini que l'objet textuel « acte de consécration » ou « inscription de consécration » (Didier MÉHU, «Le champ iconographique des images de consécration d'église »). Si l'on retient sans difficulté les images qui représentent explicitement un moment du rituel, on voit vite qu'un tel choix n'est pas suffisant. En effet, comme l'a montré Éric Palazzo ${ }^{6}$, de telles images sont très rares avant le XIII ${ }^{\mathrm{e}}$ siècle (malgré les exemples isolés du sacramentaire de Drogon, v. 830, et du pontifical de Lanaleth, vers l'an mil, qui ne peuvent s'inscrire dans une série iconographique). Pourtant, les mises en images de l'Église ne manquent pas aux temps carolingiens et grégoriens. Privilégiant rarement la représentation littérale de la liturgie, elles mettent en scène les moments fondateurs de l'Ecclesia 
(crucifixion, baptême, donation), ses médiateurs (évêques, Vierge, saints) ou la réunion de la communauté des fidèles dans le bâtiment ecclésial. La consécration d'une église est fréquemment mise en relation avec le baptême et avec la construction d'une Ecclesia régénérée, à laquelle on accède notamment par la construction (matérielle), la donation et la conversion. De la même manière que l'on ne saurait lire les commentaires liturgiques sans approfondir de telles associations, on ne peut les négliger lorsque le discours privilégie l'imago au verbum. Quelles sont alors les relations discursives, iconographiques, codicologiques entre les images du baptême, les images de l'ecclesia, les images de la construction, les images de donateurs, les images de dédicace de livres? Les réponses à ces questions nécessiteront des recherches approfondies. Pour l'heure, nous nous sommes concentrés sur quelques exemples.

Les fonts baptismaux de l'église collégiale de Freckenhorst (Westphalie) présentent une synthèse iconographique et verbale des réflexions menées au début du XII ${ }^{\mathrm{e}}$ siècle sur les implications de la consécration d'une église. C'est en effet tout autour de la cuve baptismale que l'on a gravé l'inscription qui rappelle la consécration de l'église par l'évêque Eckbert de Münster le 4 juin 1129. Le texte se développe sur un bandeau horizontal qui divise la cuve en deux parties, chacune accueillant une série d'images sculptées. En bas, un groupe de six lions entourent l'image de Daniel. En haut, sept scènes situées dans un cadre ecclésial évoquent l'Incarnation, la Passion et le salut dans un ordre chronologique qui se lit de droite à gauche. L'inscription se lit dans l'autre sens, ce qui nécessite d'effectuer un double circuit pour lire le texte et l'image. L'inscription ne commence pas sous la première scène du cycle (Annonciation), mais sous la scène du baptême du Christ, soulignant ainsi les correspondances entre cet événement fondateur et la « naissance » de l'église de Freckenhorst. La figure de Daniel située juste en dessous poursuit l'organisation verticale de l'image en inscrivant le baptême/consécration dans la série des victoires contre le mal. Le choix des sept scènes du cycle christique renforce cette impression dans la mesure où la résurrection est suivie de la descente aux limbes que l'on a préférée à la visite des saintes femmes au tombeau. On a donc affaire à une représentation articulée de l'ecclesia dans son devenir sotériologique, en insistant sur les moments essentiels de ce cheminement, tant sur le plan local (consécration) qu'universel (baptême du Christ), pour aboutir à une vision de la cité céleste où clercs et laïcs sont réunis aux pieds du Christ en gloire.

Le corpus des premiers manuscrits copiés et enluminés à Cîteaux au début du XII siècle montrent comment l'on pouvait jouer sur les correspondances figurées entre l'autel, le livre, le trône et le cadre architectural pour construire l'image d'une église vivante, productive et régénératrice (Daniel Russo, "La consécration "mise en scène" dans les images des manuscrits de Cîteaux au XII ${ }^{\mathrm{e}}$ siècle »). Si de telles images n'évoquent pas littéralement la consécration liturgique, elles construisent une représentation de l'Ecclesia fondée sur le lieu du sacrifice (autel), le siège de l'autorité ecclésiale (le trône, l'église), le verbe de Dieu (le livre), sachant que chacune de ces composantes était articulée et mise en scène lors de la liturgie quotidienne, et a fortiori lors du rituel de la consécration, véritable naissance du processus. Dans le champ monumental, une telle naissance peut être évoquée de plusieurs manières, à commencer par la mise en images des laïcs donateurs dont on ne cesse, par ailleurs, de souligner le rôle fondamental dans la fondation/consécration de l'église. Des exemples édifiants se trouvent dans les chapiteaux du chœur de Notre-Dame-du-Port à Clermont-Ferrand, de Saint-Nectaire et de l'église Saint-Priest à Volvic (Pierre-Alain MARIAux, "Images de donateurs et 
consécration d'église : exemples auvergnats autour de $1100 »)$. Les figures et les noms de quelques donateurs sont soigneusement articulés à des images de théophanie, des vertus, ou de l'Église présentée sous la forme du tabernacle de l'Ancien Testament ou d'un édifice protégé et encensé par des anges, construit de belles pierres taillées et soutenu par des colonnes massives qui abritent les signes de la présence divine figurés par l'autel et le calice. La conjonction de l'inscription et de l'image est ici particulièrement frappante. L'image de l'Église est comme portée par celle des donateurs dont l'inscription «me fecit » signale à la fois le rôle (direct ou indirect) dans la fabrication des images et celui dans la construction de l'Ecclesia.

Ces relations dialectiques nous ramènent à notre point de départ. Les commentaires du rituel de la consécration regorgent d'allusions aux species, corporea, signum, figurae, c'est-à-dire des objets, images, gestes, paroles qui vont toucher les cinq sens des participants à la consécration, véritable mysterium. Les rituels et leurs gloses, tant verbales qu'iconographiques, n'expliquent pas le mystère mais le réifient. Les jeûnes et les prières qui précèdent la cérémonie, les chants dans lesquels baigne le rituel, les circuits répétés autour de l'autel et de l'église, voire autour de l'atrium ou de la cité en portant les reliques, les mixtions d'eau, de vin, de sel et de cendre, les aspersions, les onctions et les fumigations, les signes tracés sur les tables d'autel, sur les murs et le sol des églises, puis l'insistance, lors du sermon, sur l'efficacité et les conséquences positives du rituel manifestent la réalité de l'événement exceptionnel qu'est la consécration. Les "souvenirs» gravés, peints ou verbalisés dans la pierre et le parchemin poursuivront ensuite la réification.

\section{NOTES DE FIN}

1. Étaient présents : Dominic Boulerice (Montréal), Yann Codou (Nice), Martine Clouzot (Dijon), Manuel de Souza (Saint-Étienne), Laurent Durnecker (Dijon), Marie-José GasseGrandjean (Dijon/Auxerre), Catherine Gauthier (Bruxelles), Alain Guerreau (Paris), Louis Hamilton (Toronto), Michel Lauwers (Nice), Amaury Levillayer (Paris/Québec), Eliana Magnani Soares-Christen (Dijon/Auxerre), Robert Marcoux (Québec/Dijon), Pierre-Alain Mariaux (Neuchâtel), Florian Mazel (Rennes), Didier Méhu (Québec), Alain Rauwel (Dijon), Martin Roch (Genève), Daniel Russo (Dijon), Christian Sapin (Dijon/ Auxerre), Taichiro Sugizaki (Tokyo), Cécile Treffort (Poitiers). Les actes de la table ronde seront publiés dans la collection du Centre d'Études Médiévales de Nice publiée chez Brepols, sans doute à la fin de l'année 2006.

2. Manuel de Souza prépare un article sur le champ sémantique de consecratio et dedicatio dans les œuvres de Cicéron et Tite-Live. Il sera publié dans les Actes de la table ronde d'Auxerre.

3. Un texte préalable avait été envoyé à tous les participants (D. MÉHU, « Historiae et imagines de la consécration de l'église ») dans lequel j'ai synthétisé les propos tenus lors de deux tables rondes organisées par Michel Lauwers à Nice (CEPAM) : Objets, lieux et territoires sacrés dans l'occident médiéval, 30 et 31 janvier 2004 (à laquelle ont participé Charles de Miramon, Jennifer Harris, Dominique Iogna-Prat, Michel Lauwers, Guy 
Lobrichon, Isabelle Rosé et moi-même), et La territorialisation du sacré dans l'Occident médiéval, 25 et 26 février 2005 (en compagnie de Patrick Henriet, Dominique Iogna-Prat, Michel Lauwers, Hélène Noizet, Natacha-Ingrid Tinteroff, Matthias Tischler et moimême). Parallèlement, j'ai réuni à Montréal, le 20 novembre 2004, un séminaire de recherches sur Les actes de consécration d'église au Moyen Âge central en compagnie de Jennifer Harris et Louis Hamilton. Étaient également intégrés les travaux en cours de Dominique Iogna-Prat sur la genèse d'une théorie du lieu de culte (à paraître en 2006) dont certains aspects ont été publiés : D. IOGNA-PRAT, « Lieux de culte et exégèse liturgique à l'époque carolingienne ", in C. CHAZELLE, B. VAN NAME EDWARDS (dir.), The Study of the Bible in the Carolingian Era, Turnhout, 2003, p. 215-244 ; ID., « Les moines et la "blanche robe d'églises" à l'âge roman ", in Ante el Milenario del reinado de Sancho el Mayor. Un rey navarro para España y Europa. XXX Semana de Estudios Medievales, Estella, 14 a 18 de julio de 2003, Pamplona, 2004, p. 319-347.

4. C'est ici qu'il conviendrait d'évoquer les sermons de consécration et leur tradition manuscrite qui témoigne autant du souci de formuler les implications sociales du rituel à la fin de celui-ci que de fournir un commentaire articulé susceptible de nourrir luimême les histoires, notices, inscriptions et images de la consécration. Cet aspect a été écarté de la table ronde puisqu'il avait été présenté lors de la rencontre de Nice en janvier 2004 (D. MÉHU, « Les sermons de consécration de l'église aux XI et XII ${ }^{\mathrm{e}}$ siècles »). Il sera intégré dans les Actes de la table ronde.

5. P. BUc, Dangereux rituel. De l'histoire médiévale aux sciences sociales, Paris, 2003 (éd. originale américaine, 2001).

6. É. PALAzzo, L'évêque et son image. L'illustration du pontifical au Moyen Âge, Turnhout, 1999, p. 307-356.

\section{INDEX}

Mots-clés : consécration d'église 